\title{
THE DUST, PLANETESIMALS, AND PLANETS OF HD 38529
}

\author{
Amaya Moro-Martín, ${ }^{1}$ Renu Malhotra, ${ }^{2}$ John M. Carpenter, ${ }^{3}$ Lynne A. Hillenbrand, ${ }^{3}$ Sebastian Wolf, ${ }^{4}$ \\ Michael R. Meyer, ${ }^{5}$ David Hollenbach, ${ }^{6}$ Joan Najta, ${ }^{7}$ and Thomas Henning ${ }^{4}$ \\ Received 2007 February 3; accepted 2007 June 22
}

\begin{abstract}
HD 38529 is a post-main-sequence G8 III/IV star (3.5 Gyr old) with a planetary system consisting of at least two planets having $M \sin i$ of 0.8 and $12.2 M_{\mathrm{Jup}}$, semimajor axes of 0.13 and $3.74 \mathrm{AU}$, and eccentricities of 0.25 and 0.35 , respectively. Spitzer observations show that HD 38529 has an excess emission above the stellar photosphere, with a signal-to-noise ratio $(\mathrm{S} / \mathrm{N})$ at $70 \mu \mathrm{m}$ of 4.7 , a small excess at $33 \mu \mathrm{m}(\mathrm{S} / \mathrm{N}=2.6)$, and no excess $<30 \mu \mathrm{m}$. We discuss the distribution of the potential dust-producing planetesimals from the study of the dynamical perturbations of the two known planets, considering in particular the effect of secular resonances. We identify three dynamically stable niches at $0.4-0.8,20-50$, and beyond 60 AU. We model the spectral energy distribution (SED) of HD 38529 to find out which of these niches show signs of harboring dust-producing planetesimals. The secular analysis, together with the SED modeling results, suggest that the planetesimals responsible for most of the dust emission are likely located within 20-50 AU, a configuration that resembles that of the Jovian planets + Kuiper Belt in our solar system. Finally, we place upper limits $\left(8 \times 10^{-6}\right.$ lunar masses of $10 \mu \mathrm{m}$ particles $)$ to the amount of dust that could be located in the dynamically stable region that exists between the two planets $(0.25-0.75 \mathrm{AU})$.
\end{abstract}

Subject headings: circumstellar matter — infrared: stars — Kuiper Belt — planetary systems stars: individual (HD 38529)

\section{INTRODUCTION}

HD 38529 is a post-main-sequence star (G8 III/IV) with an estimated age of $3.5 \pm 1$ Gyr (Valenti \& Fischer 2005; Gonzalez et al. 2001), a distance of $42 \pm 2$ pc (Perryman et al. 1997), and $T_{\text {eff }}=5697 \mathrm{~K}, L_{*}=6.31 L_{\odot}, M_{*}=1.47 M_{\odot}$, and $[\mathrm{Fe} / \mathrm{H}]=$ 0.445 (Valenti \& Fischer 2005). High-precision radial velocity monitoring of HD 38529 has led to the discovery of a close-in Jupiter-mass planet (HD 38529b; Fischer et al. 2001) and a second more massive and more distant planet (HD 38529c; Fischer et al. 2003). Butler et al. (2006) report the current estimates of the parameters of the two planets: masses $(M \sin i)$ of 0.8 and 12.2 $M_{\text {Jup }}$, semimajor axes of 0.13 and $3.74 \mathrm{AU}$, and eccentricities of 0.25 and 0.35 , for HD 38529 b and HD 38529c, respectively.

As part of the Spitzer Legacy Program FEPS (Formation and Evolution of Planetary Systems; Meyer et al. 2006), we searched for debris disks around the nine stars in the FEPS sample known from radial velocity studies to have one or more massive planets (Moro-Martín et al. 2007). HD 38529 was found to be the only star in that subsample to have an excess emission above the stellar photosphere, with a signal-to-noise ratio $(\mathrm{S} / \mathrm{N})$ at $70 \mu \mathrm{m}$ of 4.7, a small excess at $33 \mu \mathrm{m}(\mathrm{S} / \mathrm{N}=2.6)$, and no excess at $\lambda<30 \mu \mathrm{m}$ (Moro-Martín et al. 2007). HD 38529 therefore joined the small group of stars known to date that have both IR excess and one or more known planetary companions. Table 1 summarizes the properties of these systems, showing a wide

\footnotetext{
1 Department of Astrophysical Sciences, Princeton University, Peyton Hall, Ivy Lane, Princeton, NJ 08544; amaya@astro.princeton.edu.

${ }^{2}$ Department of Planetary Sciences, University of Arizona, 1629 East University Boulevard, Tucson, AZ 85721.

3 Department of Astronomy, California Institute of Technology, Pasadena, CA 91125.

4 Max-Planck-Institut für Astronomie, Königstuhl 17, 69117 Heidelberg, Germany.

5 Steward Observatory, University of Arizona, 933 North Cherry Avenue, Tucson, AZ 85721.

${ }^{6}$ NASA Ames Research Center, Moffet Field, CA 94035.

7 National Optical Astronomy Observatory, 950 North Cherry Avenue, Tucson AZ 85721.
}

diversity of planetary architectures. Six of these nine sources, HD 33636, HD 50554, HD 52265, HD 82943, HD 117176, and HD 128311, are similar to HD 38529 in that their Spitzer observations also show an excess at $70 \mu \mathrm{m}$ but no excess at $24 \mu \mathrm{m}$, implying that the bulk of the excess emission is arising from cool material $(T<100 \mathrm{~K}$ ) located mainly beyond $10 \mathrm{AU}$ (Beichman et al. 2005a). This means that these stars not only harbor planets (as inferred from their radial velocity observations) but also harbor an outer belt of dust-producing planetesimals (responsible for their IR excess), and in this regard they resemble the solar system in its Jovian planets + Kuiper Belt configuration. The other two sources in Table 1 (HD 69830 and $\epsilon$ Eri) have warm dust and are therefore less relevant to the present discussion.

In this paper we constrain the distribution of the potential dust-producing planetesimals from the study of the dynamical perturbations of the two known planets, considering in particular the effect of secular resonances. This allows us to identify three regions where planetesimals could be dynamically stable $(\S 2)$. After examining the lifetimes of the dust grains and concluding that the dust traces the location of the parent planetesimals $(\S 3)$, we show how we can further constrain the location of the dust (and the parent planetesimals) by modeling the HD 38529 spectral energy distribution (SED; $\S 4$ ). In $\S 5$ we discuss together the results from the dynamical and SED analysis, and finally $\S 6$ summarizes our results.

\section{POSSIBLE LOCATION OF THE DUST-PRODUCING PLANETESIMALS: EFFECT OF GRAVITATIONAL PERTURBATIONS BY THE PLANETS}

We can identify the possible location of the dust-producing planetesimals by studying the effect of the planetary perturbations on the stability of the planetesimals' orbits. Consider the orbital parameter space of semimajor axis and eccentricity, $(a, e)$. First, we can eliminate planetesimals with orbits that would cross the orbits of the planets, i.e., orbits that have either periastron or apastron within the periastron-apastron range of each of the planets. Second, we note that planetesimals in initially circular orbits 
TABLE 1

Stars with Debris Disks and Planets

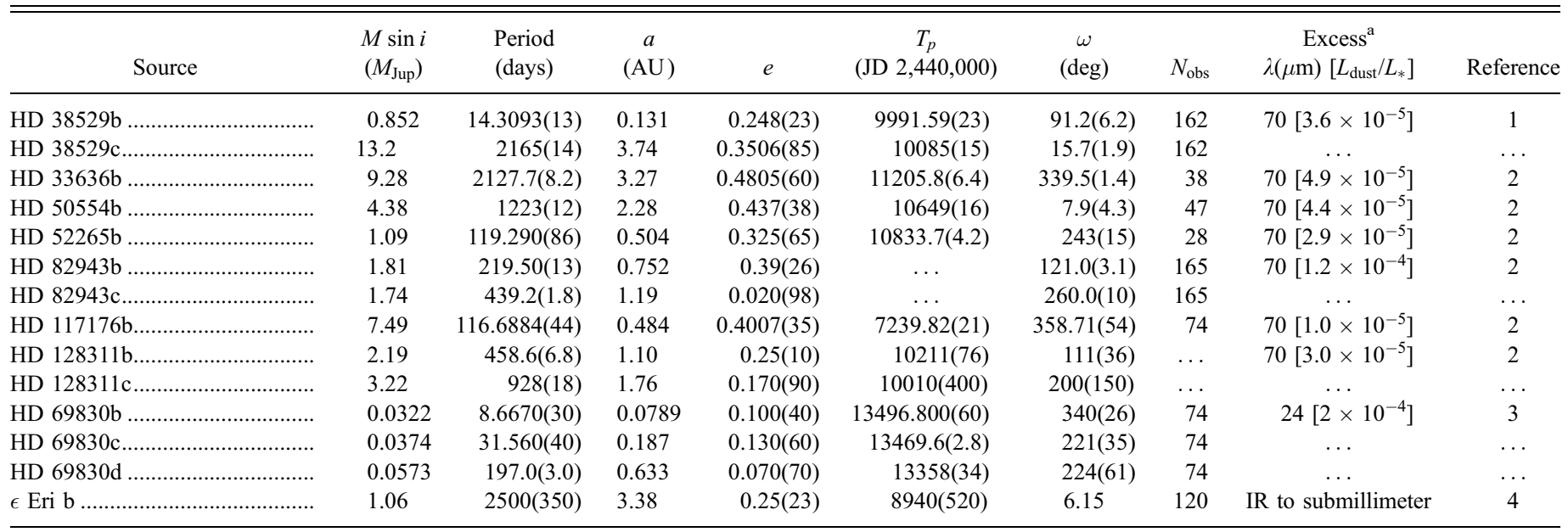

Notes.-Orbital parameters are from Butler et al. (2006): $a$ and $e$ are the semimajor axis and eccentricity of the planet, respectively, $T_{p}$ is the time of periastron passage (as a Julian day), and $\omega$ is the longitude of periastron. $N_{\mathrm{obs}}$ is the number of radial velocity observations. The numbers in parentheses indicate the uncertainty in the last significant figures.

${ }^{a}$ Wavelength at which the excess is detected, with the fractional luminosity of the excess in brackets. For stars with $70 \mu \mathrm{m}$ excess, and assuming that the peak of the emission is at $70 \mu \mathrm{m}\left(T_{\text {dust }}=52.7 \mathrm{~K}\right), L_{\text {dust }} / L_{*} \sim 10^{-5}\left(5600 / T_{*}\right)^{3}\left(F_{70, \text { dust }} / F_{70, *}\right)$, where $F_{70, \text { dust }}$ and $F_{70, *}$ are the dust excess and photospheric flux at $70 \mu \mathrm{m}$ and $T_{*}$ is the stellar temperature (Bryden et al. 2006). For HD 69830, the fractional luminosity of the excess is calculated by integrating the excess and photospheric emission between 7 and $35 \mu \mathrm{m}$.

References.-(1) Moro-Martín et al. 2007; (2) Beichman et al. 2005a; (3) Beichman et al. 2005b; (4) Greaves et al. 1998, 2005.

would be strongly unstable in the vicinity of the orbits of each of the two known planets, in a range of semimajor axis $\Delta a \simeq$ $\pm 1.5\left(m_{\text {planet }} / m_{*}\right)^{2 / 7}$ (Duncan et al. 1989). These two considerations identify several regions where planetesimals could not be stable, shown as the gray and red shaded zones in Figure 1. In addition to these perturbations that operate over short timescales, we need to consider the effect of perturbations operating over much longer timescales. These are the secular perturbations, and as we show below, they can cause a strong eccentricity excitation of the planetesimals, particularly at secular resonance locations, that can significantly shorten their lifetime.

We now calculate the effect of secular perturbations of the two planets on the planetesimals (taken as test particles on initially circular orbits). For the two planets, we assume coplanar orbits and minimum masses, taking their orbital parameters from Butler et al. (2006). For a two-planet system, there are two linear modes that excite eccentricities of test particles; we follow the LaplaceLagrange secular perturbation analysis (see Murray \& Dermott 1999). The frequencies $g_{i}$, phases $\beta_{i}$, and amplitudes $E^{(i)}$, of the two secular modes for the HD 38529 system are

$$
\begin{gathered}
g_{1}=0.106 \operatorname{arcsec} \mathrm{yr}^{-1}, \quad E^{(1)}=\left(6.697 \times 10^{-3}, 0.3300\right), \\
\beta_{1}=0.2271, \\
g_{2}=19.7 \operatorname{arcsec} \mathrm{yr}^{-1}, \quad E^{(2)}=\left(0.2787,-6.411 \times 10^{-5}\right), \\
\beta_{2}=1.594 .
\end{gathered}
$$

The secular variations of the eccentricity vectors of planets $b$, $\mathrm{c}$ are given by

$$
\begin{gathered}
e_{b} \cos \varpi_{b}=E_{1}^{(1)} \cos \left(g_{1} t+\beta_{1}\right)+E_{1}^{(2)} \cos \left(g_{2} t+\beta_{2}\right), \\
e_{b} \sin \varpi_{b}=E_{1}^{(1)} \sin \left(g_{1} t+\beta_{1}\right)+E_{1}^{(2)} \sin \left(g_{2} t+\beta_{2}\right), \\
e_{c} \cos \varpi_{c}=E_{2}^{(1)} \cos \left(g_{1} t+\beta_{1}\right)+E_{2}^{(2)} \cos \left(g_{2} t+\beta_{2}\right), \\
e_{c} \sin \varpi_{c}=E_{2}^{(1)} \sin \left(g_{1} t+\beta_{1}\right)+E_{2}^{(2)} \sin \left(g_{2} t+\beta_{2}\right) .
\end{gathered}
$$
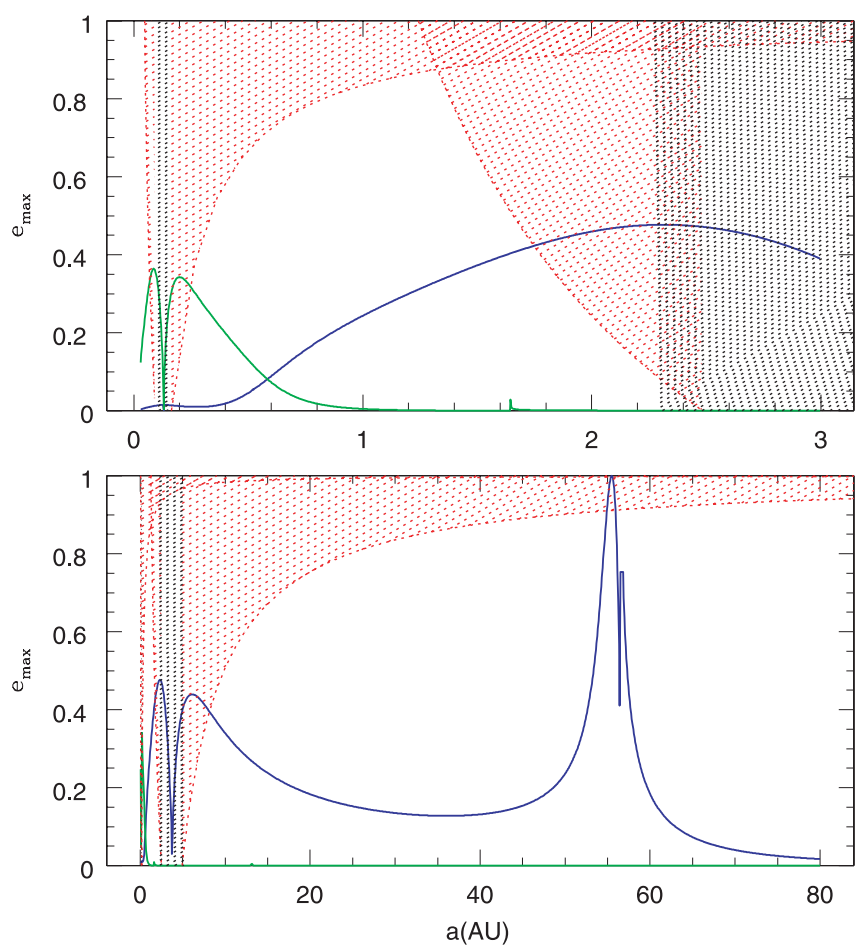

FIG. 1.- Test particle orbits that are planet-crossing (hence unstable, red shaded zone) and strongly unstable (owing to overlapping first-order mean motion resonances, gray shaded zone). The secular modes of the two planets, HD 38529b and HD 38529 c, excite the eccentricities of circular test particle orbits: the maximum eccentricity due to the fast mode is shown by the green curve, while that due to the slow mode is shown by the blue curve. The inner region, interior to the outer planet, is shown in the top panel. The bottom panel shows that the planetary perturbations are very wide ranging: secular eccentricity excitation exceeds 0.1 to nearly $60 \mathrm{AU}$; the sharp peak at $55 \mathrm{AU}$ is due to a resonance with the slow mode. 

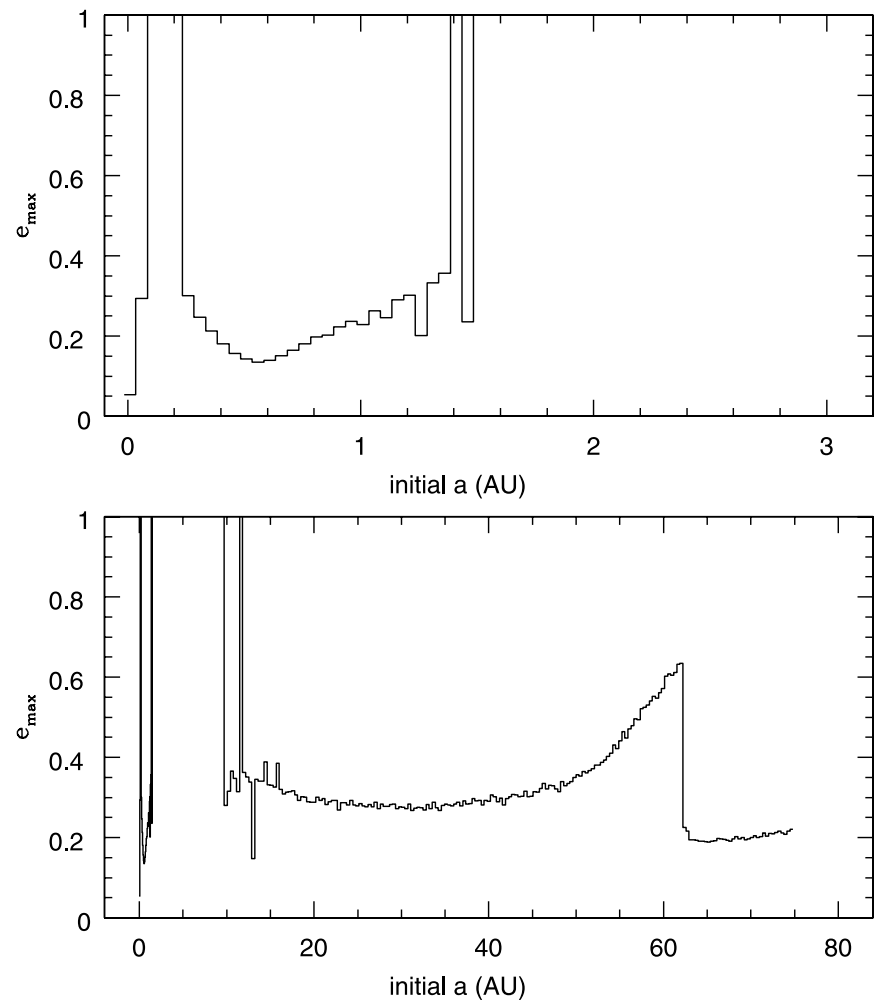

FIG. 2.-Numerical simulations of 300 test particles in the HD 38529 system of two planets. The simulations include 100 particles uniformly spaced between 0.01 and $5 \mathrm{AU}$, and 200 particles uniformly spaced between 5 and $75 \mathrm{AU}$, all in initially circular orbits coplanar with the planets. The angular elements were chosen randomly between 0 and $2 \pi$. Particles were removed if they approached the star closer than $0.005 \mathrm{AU}$ or approached a planet closer than the Hill radius of the planet. The orbits were integrated for $200 \mathrm{Myr}$ using a symplectic integrator (Wisdom \& Holman 1991). Overall, the simulations confirm the results from the secular analysis. They differ in that the maximum eccentricity in the $20-50 \mathrm{AU}$ region calculated from the secular analysis is smaller than that found in the numerical integrations; this is because the latter include secular and nonsecular perturbations (e.g., mean motion resonances), and the test particles have nonzero initial eccentricities and inclinations.

The fast mode dominates in planet b's eccentricity vector, whereas the slow mode dominates planet c's eccentricity vector.

Now consider a test particle (i.e., a planetesimal) in this system. Its secular perturbations have two components: a "free" apsidal precession due to the overall quadrupole potential (arising from the combined effects of the post-Newtonian stellar gravitational field and the gravitational effects of planetary masses approximated by uniform-density circular rings of radius equal to their semimajor axes), and a "forced" eccentricity-apsidal perturbation due to the planetary secular modes (i.e., due to the secular variations of the elliptical planetary orbits). The amplitude of the latter perturbation is a function of the semimajor axis of the test particle; at some values of the semimajor axis, where the free precession frequency is close to a planetary secular frequency, the test particle is subjected to a large-amplitude resonant eccentricity excitation. To determine the maximum forced eccentricity obtained by a test particle, we use the secular resonance analysis given in Malhotra (1998), which includes the effects of nonlinear saturation of the amplitude of the resonantly forced eccentricity from each secular mode. The results are shown in Figure 1: a test particle initially in a circular orbit would have its eccentricity excited by the slow mode to the values indicated by the blue curve; the green curve is for the eccentricity excited by the fast mode.

Clearly, very significant secular eccentricity excitation occurs over a wide zone that extends to distances much larger than the
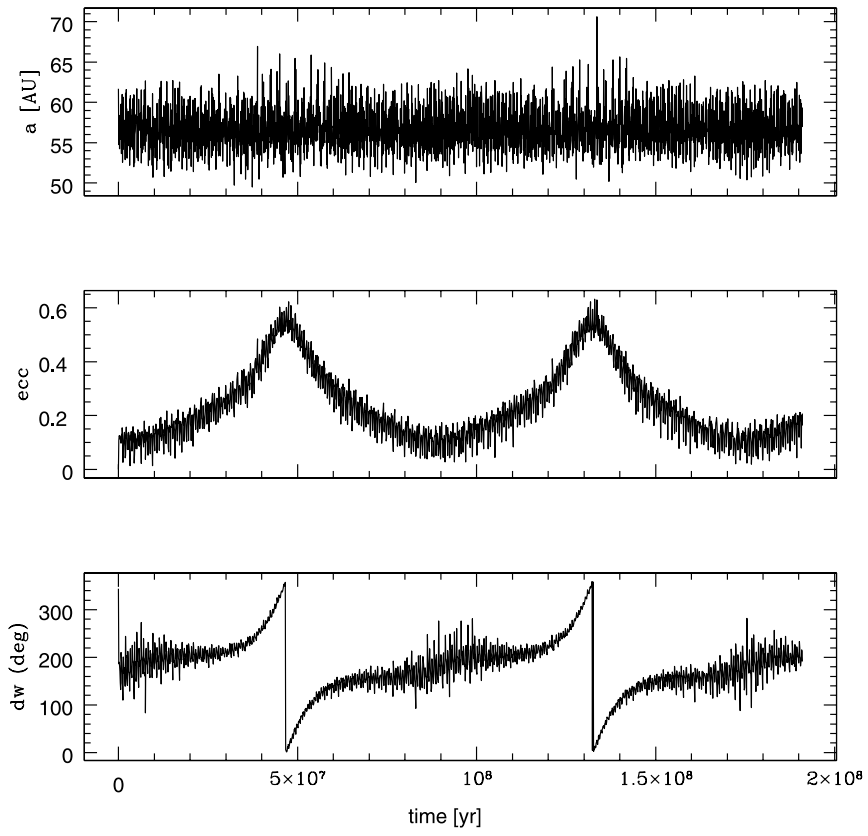

FIG. 3.- Orbital evolution over the $200 \mathrm{Myr}$ integration period of a test particle near the secular resonance. The quantity $d w$ is the difference between the longitude of periastron of the particle and that of the outermost planet. The timescale to excite the eccentricity is about $50 \mathrm{Myr}$.

unstable zones identified above. Remarkably, even though the strongly unstable zone of the outer planet extends outward to only about 5.5 AU, the eccentricity excitation exceeds 0.3 to more than $10 \mathrm{AU}$. In general, we see that the forced eccentricities exceed 0.1 everywhere up to $\sim 57 \mathrm{AU}$. A strong secular resonance with the slow mode occurs at a semimajor axis value of $\sim 55 \mathrm{AU}$, where the particles become planet-crossing or can even collide with the star; from the secular analysis (Malhotra 1998), we calculate that the timescale to excite the eccentricity from $\sim 0$ to $\sim 1$ is $\sim 1.9 \times$ $10^{8} \mathrm{yr}$. (An analogous phenomenon occurs at the inner edge of the asteroid belt in our solar system, at the location of the socalled $\nu_{6}$ secular resonance, where asteroidal apsidal precession rates are resonant with the sixth secular mode of the solar system.) Thus, tentatively, we can identify three regions that could harbor planetesimal populations (or perhaps small planets) in loweccentricity orbits: an inner region $0.4-0.8$ AU between the two planets' orbits, and two outer regions, $20-50$ and beyond $60 \mathrm{AU}$. This conclusion is confirmed by numerical integrations of test particles as demonstrated in Figures 2 and 3.

What does the high-eccentricity excitation of test particles imply for dust-producing planetesimal populations? Because these are forced eccentricities, neighboring particle orbits could have a high degree of apsidal alignment, so that the relative velocities of planetesimals would be determined by Kepler shear and therefore would be small. However, it is likely that, in the formation and evolution process, free eccentricities of planetesimal populations become comparable to the forced eccentricities, as observed in the solar system's asteroid belt and Kuiper Belt. In that case, the forced eccentricities would indeed be a measure of the relative velocities for collisions among planetesimals in these regions. Thus, if the zones identified above are populated by planetesimals, the planetary perturbations would cause mutual planetesimal collisions that would result in the production of dust.

Alternatively, because the primordial disk of HD 38529 was massive enough to form at least two giant planets, these zones arguably could harbor one or more large planetesimals, or even sub-Jovian mass planets so far undetected: sufficiently massive 
planets would tend to suppress the secularly forced eccentricities and clear out planetesimal populations from their vicinities, thereby removing sources of dust; while large $1000 \mathrm{~km}$ size planetesimals could have stirred up and ground away the planetesimal disk at early times.

\section{A COLLISION-DOMINATED SYSTEM: THE DUST TRACES THE PLANETESIMALS}

To evaluate whether the HD 38529 debris disk is radiationdominated or collision-dominated we consider the lifetimes of the dust grains.

1. The collisional lifetime at a distance $R$ from the star can be estimated as $t_{\text {col }}=P_{\text {orb }} / 8 \Sigma \sigma_{\text {geo }}$ (Backman \& Paresce 1993), where $P_{\text {orb }}$ is the orbital period, $\Sigma$ is the surface density of the grains, and $\sigma_{\text {geo }}$ is the grain geometric cross section. Here $\Sigma \sigma_{\text {geo }}$ is the dimensionless fractional surface area of the disk, of the order of $L_{\text {dust }} / L_{*} \sim 10^{-5}\left(5600 / T_{*}\right)^{3}\left(F_{70, \text { dust }} / F_{70, *}\right)$ (Backman \& Paresce $1993)$. For HD $38529, F_{70, *}=17.4 \mathrm{mJy}, F_{70, \text { dust }}=66.9 \mathrm{mJy}$, $T_{*}=5697 \mathrm{~K}$, and $M_{*}=1.47 M_{\odot}$ (Moro-Martín et al. 2007), so that $L_{\text {dust }} / L_{*}=3.6 \times 10^{-5}$ and

$$
t_{\text {col }}=3.4 \times 10^{3} P_{\text {orb }}=2.8 \times 10^{3}\left(\frac{R}{\mathrm{AU}}\right)^{3 / 2} \mathrm{yr} .
$$

2. The Poynting-Robertson (P-R) lifetime at distance $R$, i.e., the time it takes for the dust grain to migrate from $R$ to the star, is given by (Burns et al. 1979; Backman \& Paresce 1993)

$$
\begin{aligned}
t_{\mathrm{P}-\mathrm{R}} & =\frac{4 \pi b \rho}{3} \frac{c^{2} R^{2}}{L_{*}} \\
& =710\left(\frac{b}{\mu \mathrm{m}}\right)\left(\frac{\rho}{\mathrm{g} \mathrm{cm}^{-3}}\right)\left(\frac{R}{\mathrm{AU}}\right)^{2}\left(\frac{L_{\odot}}{L_{*}}\right) \frac{1}{1+\text { albedo }} \mathrm{yr},
\end{aligned}
$$

where $b$ and $\rho$ are the grain radius and density, respectively. More relevant is the time it takes for the particle to drift under P-R drag from a populated to a relatively unpopulated region, i.e., the time it would take to fill out the gap under P-R drag $\left(t_{\mathrm{P}-\mathrm{R}}^{\text {fill }}\right)$. If the scale over which the dust density significantly decreases is $x \%$ of $R$, this time is

$$
t_{\mathrm{P}-\mathrm{R}}^{\mathrm{fill}}=\left[1-\left(1-\frac{x}{100}\right)^{2}\right] t_{\mathrm{P}-\mathrm{R}}=2.0 \times 10^{4}\left(\frac{R}{\mathrm{AU}}\right)^{2} \mathrm{yr},
$$

where we have assumed that $x=10, L_{*}=6.3 L_{\odot}, \rho=2.5 \mathrm{~g} \mathrm{~cm}^{-3}$, $b=10 \mu \mathrm{m}$, and albedo $=0.1$.

If the sources of dust are outside the orbit of the planet, and if P-R drag dominates the dynamics, as the dust particles drift inward due to P-R drag they are likely to be scattered out of the system when crossing the orbit of a planet, creating a dustdepleted region inside the planet's orbit (Roques et al. 1994; Liou \& Zook 1999; Moro-Martín \& Malhotra 2002, 2003). The ejection is very efficient for planets in circular orbits: planets with masses of 3-10 $M_{\text {Jup }}$ located between 1 and 30 AU eject $>90 \%$ of the particles that go past their orbits, while a $1 M_{\text {Jup }}$ planet at $30 \mathrm{AU}$ ejects $>80 \%$ of the particles and about $50 \%-$ $90 \%$ if located at 1 AU (Moro-Martín \& Malhotra 2005); these results are valid for dust particle sizes in the range $0.7-135 \mu \mathrm{m}$ (assuming astronomical silicate composition) and when the central star is solar-type.
On the other hand, if the system is collision-dominated (Krivov et al. 2000; Dominik \& Decin 2003; Wyatt 2005), mutual collisions can fragment the larger particles to smaller and smaller sizes, until they are blown out from the system by radiation pressure. This means that the dust particles would be destroyed before they migrate far from the location of their parent bodies under P-R drag; i.e., the dust traces the location of the parent bodies. In this case, an inner cavity in the dust density distribution could only arise if the planetesimals themselves are confined to a belt, i.e., if there is an inner edge to their spatial distribution. This scenario may suggest a massive planet confining the inner edge of the dust-producing planetesimals.

For the HD 38529 system, the estimates in equations (7) and (9) suggest that $t_{\mathrm{col}} \ll t_{\mathrm{P}-\mathrm{R}}^{\text {fill }}$ at all relevant radii; i.e., we are in the collision-dominated regime. This means that the dust emission traces the location of the dust-producing planetesimals, with dynamically stable niches at $0.4-0.8,20-50$, and beyond $60 \mathrm{AU}$. To find out which of these niches do actually show signs of harboring dust-producing planetesimals we turn now to the study of the IR excess emission detected by Spitzer.

\section{SED MODELING}

\subsection{Single-Temperature Models are Insufficient}

The $70 \mu \mathrm{m}$ observations of HD 38529 are not spatially resolved, and therefore it is not possible to know unambiguously the spatial distribution of the dust. We can learn about the characteristic temperature of the dust from the spectral energy distribution (L. A. Hillenbrand et al. 2007, in preparation). From the ratio of the excess emission at $70 \mu \mathrm{m}$ to that at $33 \mu \mathrm{m}$, and assuming these wavelengths are at the Wien's tail of the dust blackbody emission, the ratio of the excess fluxes yields a characteristic temperature of $43 \pm 4 \mathrm{~K}$. If the blackbody grains are in thermal equilibrium, we can calculate the location of the dust from (Backman \& Paresce. 1993)

$$
\frac{R_{\text {dust }}}{\mathrm{AU}}=\left(\frac{L_{*}}{L_{\odot}}\right)^{1 / 2}\left(\frac{278 \mathrm{~K}}{T_{\text {dust }}}\right)^{2}
$$

for HD $38529, R_{\text {dust }}=106 \pm 18$ AU. The dust mass can then be estimated by assuming the dust is in a thin shell at a distance $R_{\text {dust }}$, and that the grains are spherical with cross section equal to their geometric cross section, so that the total number of dust particles is $\sim\left(4 \pi R_{\text {dust }}^{2} / \pi b^{2}\right) \tau$ and $M_{\text {dust }} \sim 16 \pi R_{\text {dust }}^{2} \tau b \rho / 3$. The optical depth, $\tau$, can be approximated as $L_{\text {dust }} / L_{*}$ so that (Jura et al. 1995)

$$
\frac{M_{\text {dust }}}{M_{\oplus}} \sim 6.28 \times 10^{-5}\left(\frac{L_{\text {dust }}}{L_{*}}\right)\left(\frac{\rho}{\mathrm{g} \mathrm{cm}^{-3}}\right)\left(\frac{\langle b\rangle}{\mu \mathrm{m}}\right)\left(\frac{R_{\text {dust }}}{\mathrm{AU}}\right)^{2},
$$

where $b$ and $\rho$ are the particle's radius and density. It is important to note that the above estimate is a lower limit: in the absence of sensitive (sub)millimeter detections, no realistic constraints can be made to the dust mass, a significant fraction of which could be locked in grains with sizes of $\sim 1 \mathrm{~mm}$ that emit efficiently in the (sub)millimeter but contribute little to the infrared emission. For HD 38529, $L_{\text {dust }} / L_{*}=3.6 \times 10^{-5}$, and using $b=10 \mu \mathrm{m}$ and $\rho=$ $2.5 \mathrm{~g} \mathrm{~cm}^{-3}$ we obtain $M_{\text {dust }}>1.9 \times 10^{-9} M_{\odot}{ }^{8}{ }^{8}$

\footnotetext{
8 Dust mass estimates for the Kuiper Belt dust disk range from a total dust mass $<3 \times 10^{-10} M_{\odot}$ (Backman et al. 1995) to $\sim 4 \times 10^{-11} M_{\odot}$ for dust particles $<150 \mu \mathrm{m}$ (Moro-Martín \& Malhotra 2003), with a fractional luminosity of $L_{\text {dust }} / L_{*} \sim 10^{-7}$ to $10^{-6}$ (Stern 1996). The fractional luminosity of the asteroid belt dust (zodiacal cloud) is estimated to be $L_{\text {dust }} / L_{*} \sim 10^{-8}$ to $10^{-7}$ (Dermott et al. 2002).
} 
Another estimate of the dust temperature can be made from fitting a photosphere plus a single-temperature blackbody to the IRS spectrum only; this results in a dust temperature of $79 \mathrm{~K}$, which would correspond to a distance of 31 AU. The discrepancy with the characteristic temperature derived from the ratio of the excess emission at $70 \mu \mathrm{m}$ to that at $33 \mu \mathrm{m}$ indicates that it is not possible to conclude that the dust is confined to a narrow ring as implied by the assumption of a single grain temperature. Similarly, L. A. Hillenbrand et al. (2007, in preparation) find that the SEDs of about one-third of the FEPS targets with $70 \mu$ m excess emission are better fit by multitemperature rather than singletemperature blackbody models. The need for a multitemperature grain distribution has previously been found in systems such as $\beta$ Pic (e.g., Li \& Greenberg 1998; Telesco et al. 2005) and HR 4796 A (e.g., Li \& Lunine 2003) and has been unambiguously confirmed by the eight spatially resolved observations in scattered light of debris disks known to date, which led Kalas et al. (2006) to conclude that debris disks show two basic architectures: (1) belts about 20-30 AU wide and with well-defined outer boundaries (HR 4796 A, Fomalhaut, and HD 139664), and (2) wider belts with sensitivity-limited edges implying widths >50 AU (HD 32297, $\beta$ Pic, AU Mic, HD 107146, and HD 53143). In the absence of spatially resolved observations, the SED modeling of HD 38529 (or any other source under consideration) should enable exploration of a range of disk architectures.

\subsection{Multitemperature (SED) Models}

\subsubsection{SED Modeling Assumptions}

For the modeling of the SED, we use the radiative transfer code developed by Wolf \& Hillenbrand (2003). Because of the above considerations, we model the dust disk as an annulus of inner radius $R_{\text {in }}$, outer radius $R_{\text {out }}$, total dust mass $M_{\text {dust }}$, and a constant surface density $\left[\Sigma \propto r^{0}\right.$, so that the number density $\left.n(r) \propto r^{-1}\right]$. We assume that the dust grains are composed of silicates ${ }^{9}$ with optical constants from Weingartner \& Draine (2001). For the particle sizes we consider two options: (1) a single grain size of $10 \mu \mathrm{m}$ in radius and (2) a particle size distribution following a power law, $n(b) \propto b^{-q}$, where $b$ is the particle radius, $q=3.5$ (for grains in collisional equilibrium), $b_{\min }=2 \mu \mathrm{m}$, and $b_{\max }=10 \mu \mathrm{m}$. In both cases, the radius of $10 \mu \mathrm{m}$ was chosen because such a grain radiates efficiently at $70 \mu \mathrm{m}$. Because of the last property, this choice of grain size provides a lower limit for $M_{\text {dust }}$. The value selected for $b_{\text {min }}$ corresponds to the "blow out" size, i.e., the minimum size of the grains that can remain bound in the system, given by (Artymowicz et al. 1989)

$$
\frac{b_{\min }}{\mu \mathrm{m}}=0.52 \frac{2.5 \mathrm{~g} \mathrm{~cm}^{-3}}{\rho} \frac{1+\text { albedo }}{1.1} \frac{L_{*} / L_{\odot}}{\left(M_{*} / M_{\odot}\right)\left(T_{*} / 5780 \mathrm{~K}\right)} .
$$

For HD 38529, $L_{*}=6.31 L_{\odot}, M_{*}=1.47 M_{\odot}$, and $T_{*}=5697 \mathrm{~K}$; using a grain density $\rho=2.5 \mathrm{~g} \mathrm{~cm}^{-3}$ and albedo $=0.1$, we get $b_{\min }=2 \mu \mathrm{m}$.

We assume that the dust disk is optically thin with the dust being in thermal equilibrium with the stellar radiation field. Only scattering, absorption, and reemission of stellar radiation by dust grains are taken into account, neglecting scattering and dust heating from the infrared radiation produced by the optically thin dust disk. With these assumptions, the temperature of the dust

\footnotetext{
9 For a study on how the SEDs depend on the grain composition we refer to Wolf \& Hillenbrand (2003) and Moro-Martín et al. (2005).
}

grain, for a given dust size and a chemical composition, depends only on the distance to the central star. ${ }^{10}$

The outer radius of the disk, $R_{\text {out }}$, cannot be constrained with data currently available. Based on scattered-light observations from nearby debris disks, disk sizes of about a hundred to several hundred AU are inferred (Kalas et al. 2005; Dent et al. 2000; Greaves et al. 2000; Wilner et al. 2002; Holland et al. 2003; Liu 2004; Metchev et al. 2005; Ardila et al. 2004). For this reason we consider disk sizes of $R_{\text {out }}=50 \mathrm{AU}$ (solar system size), $R_{\text {out }}=$ $100 \mathrm{AU}$, and $R_{\text {out }}=500 \mathrm{AU}$.

With the above three values for $R_{\text {out }}$, assuming a uniform density distribution, and with the grain size and composition fixed, we then vary $R_{\text {in }}$ and $M_{\text {dust }}$ (our only two free parameters) to create a grid of models where we allow $R_{\text {in }}$ to vary from the silicate sublimation radius $\left(R_{\text {sub }}\right.$, where $\left.T_{\text {sub }}=1550 \mathrm{~K}\right)$ to $R_{\text {out }}$. This accounts for the possibility of having either a dust disk of wide radial extent or a narrow ring of dust.

The observations to be modeled are the Spitzer IRS ${ }^{11}$ synthetic photometric points at 13, 24, and $33 \mu \mathrm{m}$ and the Spitzer MIPS photometric points at 24 and $70 \mu \mathrm{m}$ (from Moro-Martín et al. 2007). To evaluate whether a particular model is a valid fit to these data points, or if not, to what degree of certainty the model can be excluded, we calculate its $\chi^{2}$ probability, $P\left(\chi^{2} \mid \nu\right)$, where $\nu$ is the number of degrees of freedom. In our case $\nu=2$, as the only two free parameters are $R_{\text {in }}$ and $M_{\text {dust }}$, and all the other disk parameters and dust properties are fixed to the values given above. The probability is defined so that $P(0 \mid \nu)=0$ and $P(1 \mid \nu)=1$. Models with $P\left(\chi^{2} \mid \nu\right)>0.9973$ can be excluded with a $3 \sigma$ certainty, while models with $P\left(\chi^{2} \mid \nu\right)>0.683$ could be excluded with a $1 \sigma$ certainty.

\subsubsection{SED Modeling Results}

Figure 4 shows the two-dimensional grids of SED models described above: green points show the models with $P\left(\chi^{2} \mid \nu\right)<$ 0.683 , i.e., models that are consistent with the observations; red points represent models with $P\left(\chi^{2} \mid \nu\right)>0.683$, i.e., models that could be excluded with a $1 \sigma$ certainty; and black points correspond to models with $P\left(\chi^{2} \mid \nu\right)>0.9973$, i.e., models that can be excluded with a $3 \sigma$ certainty. Some of these SEDs are shown in Figure 5 together with the observations. As can be seen in both figures, the models are degenerate and for a given $R_{\text {out }}$, there are many pairs of $R_{\text {in }}$ and $M_{\text {dust }}$ that could fit the observed SED. Because of this degeneracy, our main interest lies in identifying the models that can be excluded. These are of particular interest because they allow us to identify dust-depleted regions

\footnotetext{
${ }^{10}$ Our SED modeling assumes that the observed dust excess arises from a circumstellar disk. However, the outermost planet has a $M \sin i$ of $12.2 M_{\text {Jup }}$, placing it in the boundary between planets and brown dwarfs. Even though the latter could potentially harbor a disk, the prevalence of cold dust in the HD 38529 system indicates that this could not be the dominant source of the observed dust because this disk would be located near the star (the outermost planet's semimajor axis is 3.74 AU) implying the presence of warm dust that is not observed.

${ }^{11}$ The IRS spectrum for $\lambda<14.21 \mu \mathrm{m}$ (short-low module) shows a small offset with respect to the Kurucz model. Because the emission at those wavelengths is clearly photospheric, we have multiplied the spectrum for $\lambda<14.21 \mu \mathrm{m}$ by 1.045 to make it coincide with the stellar photosphere (so these wavelengths do not dominate the $\chi^{2}$ statistics). In addition, the IRS spectrum shows a small discontinuity at $14.21 \mu \mathrm{m}$, possibly because the star was not centered in the slit of the long-low module. To correct for this discontinuity, we have multiplied the IRS spectrum for $\lambda>14.21 \mu \mathrm{m}$ by 1.108 . Accounting for this, the corrected $33 \mu \mathrm{m}$ flux is $95 \pm 2 \mathrm{mJy}$, where the error is the internal uncertainty. For the calculation of the $\mathrm{S} / \mathrm{N}$ of the excess we used the total uncertainty, obtained from adding in quadrature the internal uncertainty and the calibration uncertainty, the latter taken to be $6 \%$. This is an underestimate of the significance of the departure from a pure photosphere because this calibration uncertainty is for the overall spectrum, not for individual wavelengths relative to each other.
} 

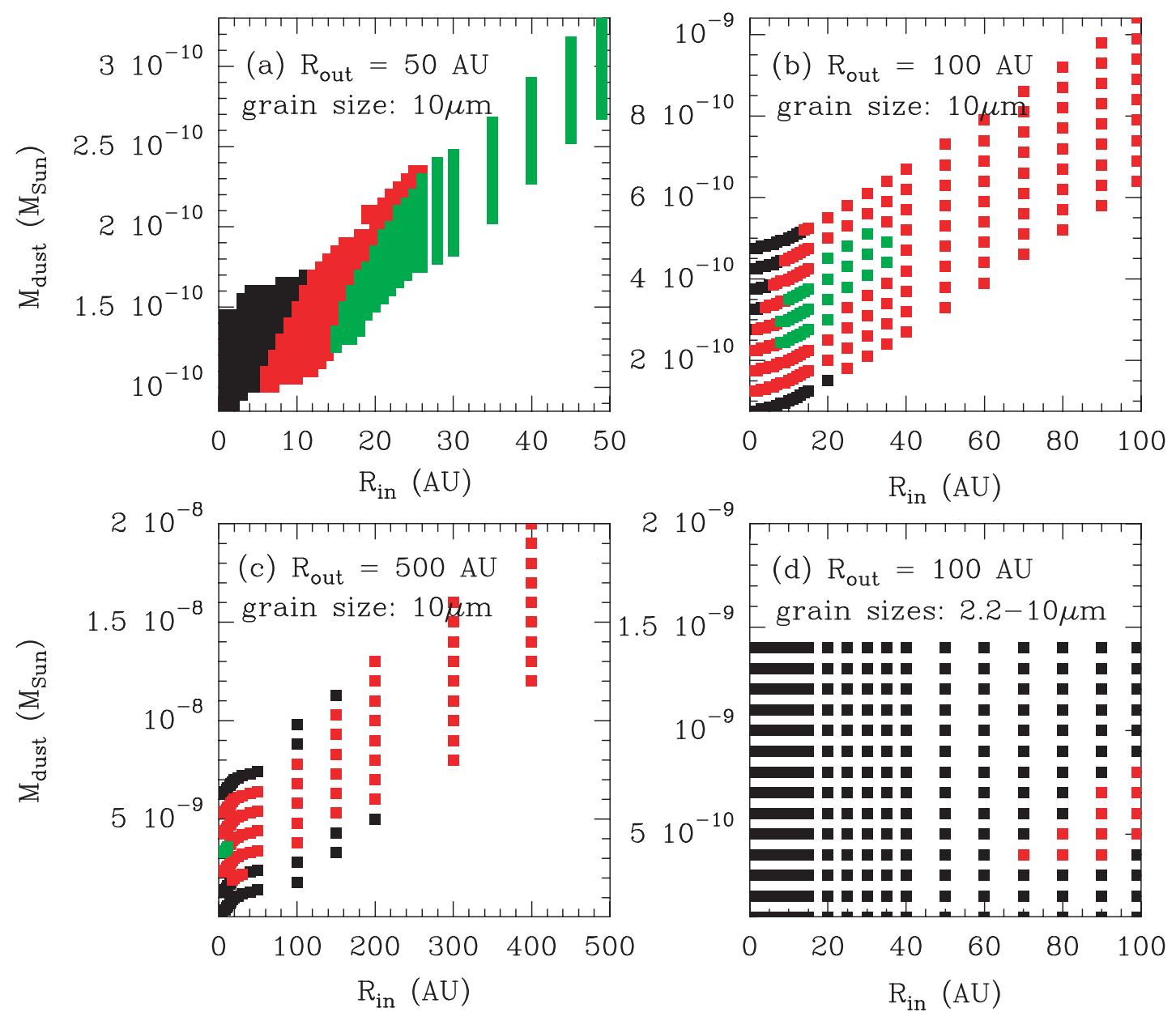

FIG. 4.- Two-dimensional grids where each point represents a modeled SED, where $R_{\text {in }}$ and $M_{\text {dust }}$ are the two free parameters. (a) $-(c)$ Models with a single grain size of $10 \mu \mathrm{m}$ and with $R_{\text {out }}=50,100$, and $500 \mathrm{AU}$, respectively. $(d)$ Models with a distribution of grain sizes given by $n(b) \propto b^{-3.5}$, with $b_{\min }=2 \mu \mathrm{m}$ and $b_{\max }=10 \mu \mathrm{m}$. The green points show models with $P\left(\chi^{2} \mid \nu\right)<0.683$, red points in $(a)-(c)$ represent models with $P\left(\chi^{2} \mid \nu\right)>0.683$, i.e., models that can be excluded with $1 \sigma$ certainty, and black points correspond to models with $P\left(\chi^{2} \mid \nu\right)>0.9973$, i.e., models that are excluded with $3 \sigma$ certainty. The red points in $(d)$ have $P\left(\chi^{2} \mid \nu\right)>0.988$; i.e., they are all close to being excluded with a $3 \sigma$ certainty.

whose origin can be studied in terms of the overall dynamics of the planetary system (see $\S 2)$.

The main results from Figures $4 a-4 c$ regarding the models that assume grains $10 \mu \mathrm{m}$ in radius are the following.

1. For $R_{\text {out }}=50 \mathrm{AU}$, we can exclude models with $R_{\text {in }} \lesssim 5 \mathrm{AU}$ with a certainty of $3 \sigma$, or we could exclude models with $R_{\text {in }} \lesssim$ 14 AU with a certainty of $1 \sigma$; the data are consistent with models having $15 \mathrm{AU} \lesssim R_{\text {in }} \lesssim 50 \mathrm{AU}$, i.e., models ranging from a $35 \mathrm{AU}$ wide disk (extending from 15 to $50 \mathrm{AU}$ ) to a very narrow ring of dust located at $50 \mathrm{AU}$. In other words, the observations require the presence of an inner cavity in the dust density distribution of at least $5 \mathrm{AU}$ in radius, with larger cavities consistent with the data (the latter implying narrower rings of larger dust mass).

2 . For $R_{\mathrm{out}}=100 \mathrm{AU}$, none of the $R_{\text {in }}$ considered (ranging from the dust sublimation radius, $R_{\text {sub }}$, to $100 \mathrm{AU}$ ) can be excluded with a $3 \sigma$ certainty; while models with $R_{\text {in }} \lesssim 7$ AU and those with $R_{\text {in }} \gtrsim 40$ AU could be excluded to $1 \sigma$. Possible fits include models with 8 AU $\lesssim R_{\text {in }} \lesssim 35$ AU.

3. For $R_{\text {out }}=500 \mathrm{AU}$, again none of the $R_{\text {in }}$ considered (from $R_{\text {sub }}$ to $400 \mathrm{AU}$ ) can be excluded with a $3 \sigma$ certainty; while models with $R_{\text {in }} \gtrsim 14$ AU could be excluded to $1 \sigma$. Possible models include those with $R_{\text {sub }} \lesssim R_{\text {in }} \lesssim 12$ AU.

We can understand these results as follows. For a constant surface density, $\Sigma \propto r^{0}$, there is 100 times more dust mass between
50 and $100 \mathrm{AU}$ than there is between 5 and $10 \mathrm{AU}$, while the dust temperature is proportional to $r^{-1 / 2}$. The result is that there is a significant contribution to the $70 \mu \mathrm{m}$ emission from the largest radii, $R_{\text {out }}$. To keep the $70 \mu \mathrm{m}$ emission constant, the dust surface area or mass at $R_{\text {out }}$ must be similar as $R_{\text {out }}$ varies, i.e., $\Sigma R_{\text {out }} \sim$ constant, which means that: (1) larger $R_{\text {out }}$ requires a smaller $\Sigma$, and smaller $\Sigma$ means that we can tolerate smaller inner radii since there is now less dust mass placed there, which explains why the SEDs can be fitted with a smaller inner cavity for $R_{\text {out }}=100 \mathrm{AU}$ and with a constant surface density all the way to the sublimation radius in the $500 \mathrm{AU}$ case; and (2) smaller $R_{\text {out }}$ requires a larger $\Sigma$, and in order to keep the 24 and $33 \mu$ m fluxes below their upper limits, this requires an increase in the inner cavity size. A larger inner cavity would also be needed if we had assumed $\Sigma \propto r^{-1}$ instead of constant.

In the models shown in Figure $4 d$ we relax the assumption that all the grains are $10 \mu \mathrm{m}$ in radius and allow for the presence of smaller grains, with the particle size distribution following $n(b) \propto$ $b^{-3.5}$, and with $b_{\min }=2 \mu \mathrm{m}$ and $b_{\max }=10 \mu \mathrm{m}$. We find that most of the models can be excluded with a $3 \sigma$ certainty; i.e., we can exclude the presence of a significant population of small grains inside $100 \mathrm{AU}$ based on the lack of a significant continuum emission at $\lambda<30 \mu \mathrm{m}$. A similar conclusion has been obtained from several other debris disks whose spectroscopy shows little or no solid state features, indicating that the dust grains 


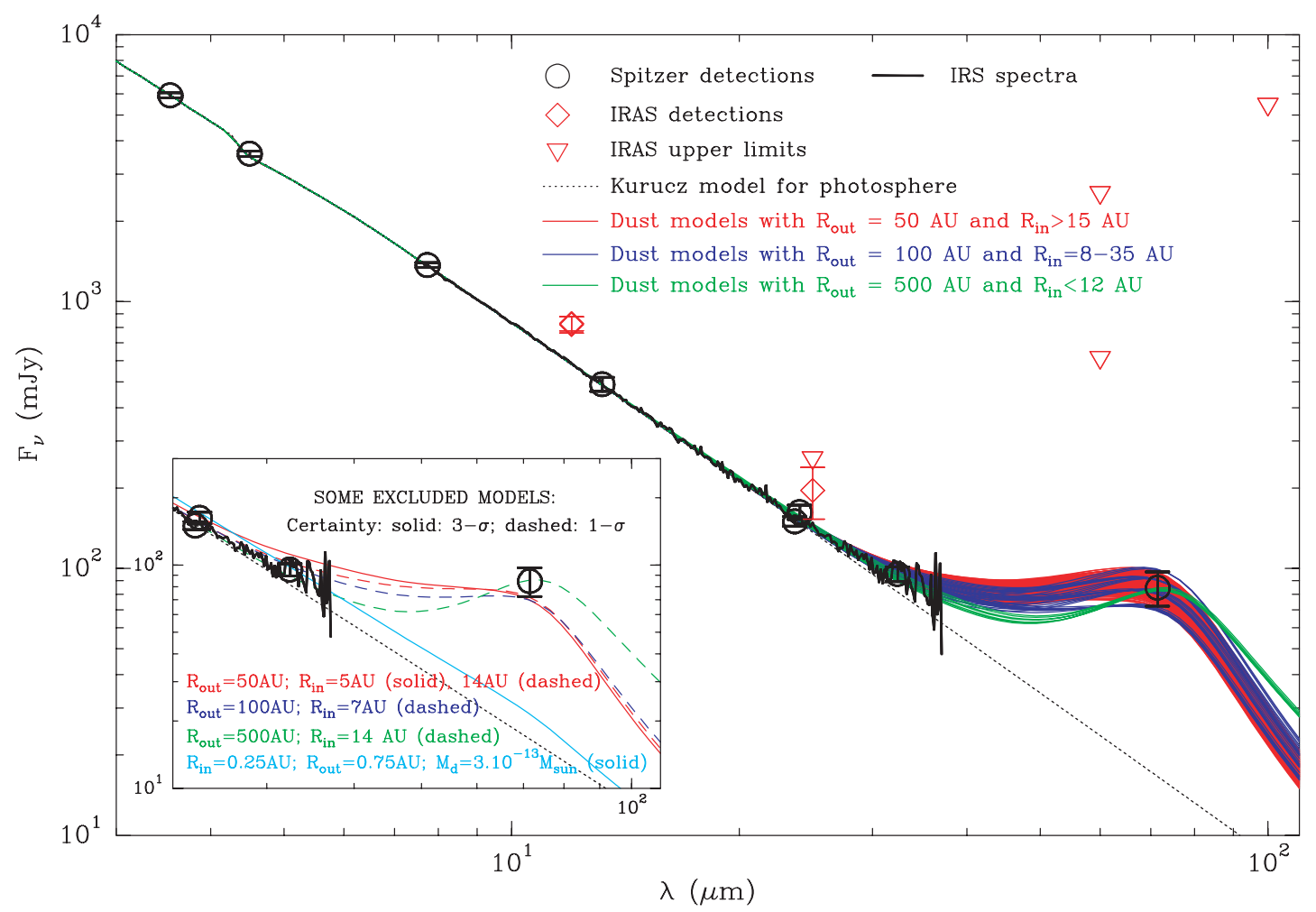

FIG. 5.- Observed and modeled SEDs for HD 38529. The black dotted line is the Kurucz model. The black thick solid line is the IRS low-resolution spectrum. The photometric points are identified as follows: black circles are the new Spitzer observations (IRAC, MIPS, and synthetic photometry from IRS); red diamonds are IRAS observations. In all cases, the error bars correspond to $1 \sigma$ uncertainties. Upper limits are represented by triangles and are given when $F / \Delta F<3$ and placed at $F+3 \Delta F$ if $F>0$ or $3 \Delta F$ if $F<0$. The colored solid lines in the main panel show three sets of models that fit the observations with a $\chi^{2}$ probability $<0.68$ (corresponding to the models represented by green points in Fig. 4). The models include the emission from the photosphere and from a dust disk composed of astronomical silicate grains $10 \mu \mathrm{m}$ in radius. We assume the dust disk extends from $R_{\text {in }}$ to $R_{\text {out }}$ with a constant surface density. We consider three values for $R_{\text {out }}: 50 \mathrm{AU}$ (red), $100 \mathrm{AU}$ (blue), and $500 \mathrm{AU}$ (green); $R_{\text {in }}$ and $M_{\text {dust }}$ are allowed to vary. The inset at the lower left shows the most relevant excluded models. The solid line represents models excluded with a certainty of $3 \sigma$, while the dashed line corresponds to $1 \sigma$. The model shown in light blue gives an upper limit to the amount of warm dust located between 0.25 and $0.75 \mathrm{AU}$.

have sizes $\gtrsim 10 \mu \mathrm{m}$ (e.g., Jura et al. 2004; Stapelfeldt et al. 2004).

\section{DISCUSSION OF THE DYNAMICAL AND THE SED ANALYSIS}

\subsection{Location of Cold Dust}

The two outermost regions identified in $\S 2$ where planetesimals could survive for extended periods of time in the presence of the two known radial velocity detected planets, namely, 20-50 and beyond $60 \mathrm{AU}$, are in broad agreement with the allowed dust locations that result from the modeling of the SED. In particular, we saw that the models with $R_{\text {out }}=50 \mathrm{AU}$ predicted an inner cavity of radius $>5 \mathrm{AU}$, while a range of dust disks with $R_{\text {out }}=$ $50 \mathrm{AU}$ and $R_{\text {in }}>20 \mathrm{AU}$ could fit the observed SED. From the models with $R_{\text {out }}=100 \mathrm{AU}$, we can conclude that it is very unlikely that the observed dust emission arises only from planetesimals located beyond $60 \mathrm{AU}$ because the models with $R_{\text {out }}=100 \mathrm{AU}$ and $R_{\text {in }}=60,70,80$, and 90 AU have $P\left(\chi^{2} \mid \nu\right)$ larger than $0.83,0.87$, 0.89 , and 0.91 , respectively A similar conclusion can be drawn from the models with $R_{\text {out }}=500 \mathrm{AU}$ and $R_{\text {in }}>60 \mathrm{AU}$, for which $P\left(\chi^{2} \mid \nu\right)>0.94$. Therefore, from the dynamical and SED modeling we conclude that the planetesimals responsible for most of the dust emission are likely located within the 20-50 AU region.

Given that the maximum eccentricities in the 20-50 AU region are moderately high, $0.25-0.3$ (see Figs. 1 and 2) - implying collisional velocities at $30 \mathrm{AU}$ of approximately $0.3 v_{\text {circ }}=2.8 \mathrm{~km} \mathrm{~s}^{-1}$ - could a moderate rate of dust production persist in this relatively old system? (A "moderate rate" is important in order for the dust production to be long-lived, instead of being depleted rapidly in the early history of the system.) Following Wyatt et al. (2007), we can estimate the maximum fractional luminosity of the excess, $f_{\max }$, that could originate from a planetesimal belt of a given age that is evolving in a quasi-steady state. Wyatt's analytical model assumes that the planetesimals and the dust are in collisional equilibrium and that their size distribution follows a continuous power law of index -3.5 . For HD 38529, and given the results above, we assume that the planetesimal belt extends from 20 to $50 \mathrm{AU}$, with a mean planetesimal eccentricity of 0.3 . Other parameters in the model are the diameter of the largest planetesimal in the cascade, $2000 \mathrm{~km}$, and the specific incident energy required to catastrophically destroy a planetesimal, $200 \mathrm{~J} \mathrm{~kg}^{-1}$. At the age of the $\mathrm{HD}$ 38529 system, $3.5 \mathrm{Gyr}$, the model predicts $f_{\max }=2.26 \times 10^{-6}$ (see eq. [20] in Wyatt et al. 2007). This value is smaller than the observed fractional luminosity of the excess, $f_{\text {obs }}=3.6 \times 10^{-5}$ (see $\S 3$ ). However, because there is an uncertainty of 2 orders of magnitude in the estimate of $f_{\max }$, we cannot reject a scenario in which the dust observed is the result of the steady grinding down of planetesimals. If we were to assume that the above value of $f_{\max }$ is correct, an estimate of the timescale over which the fractional luminosity can be maintained at the value of $f_{\text {obs }}$ is given by $t_{\text {age }} f_{\text {max }} / f_{\text {obs }}=220 \mathrm{Myr}$. In this case, a possible scenario could be that the stable region beyond $60 \mathrm{AU}$ supplies some planetesimals that drift into the $20-50 \mathrm{AU}$ region by nongravitational effects. 


\subsection{Upper Limits on Warm Dust}

In $\S 2$ we identified a modestly stable small zone between the two known planets (0.4-0.8 AU), which has also been identified in numerical simulations by Barnes \& Raymond (2004); our secular perturbation analysis provides a theoretical explanation for those numerical stability results. Raymond \& Barnes (2006) consider terrestrial planet accretion in this zone. They conclude that HD 38529 is likely to support an asteroid belt and perhaps Marssized planets, but not larger planets, because the potential feeding zone for the accretion of a terrestrial planet would be limited by the high eccentricities of the planetesimals in this region. The lack of an IR excess at wavelengths shorter than $30 \mu \mathrm{m}$ allows us to place an upper limit on the amount of warm (asteroidal) dust that could be located in this inner region. We use the IRAC 5 and $8 \mu \mathrm{m}$, IRS $13 \mu \mathrm{m}$, and MIPS $24 \mu \mathrm{m}$ photometric measurements, and we assume $10 \mu \mathrm{m}$ size silicate grains (optical constants taken from Weingartner \& Draine 2001). We find that a $3 \sigma$ upper limit to the dust mass in this potential asteroid belt is $3 \times 10^{-13} M_{\odot}$ or $10^{-7} M_{\oplus}$. For comparison, the mass estimate for the zodiacal cloud in the terrestrial planet region of the solar system is $3 \times$ $10^{-10} M_{\oplus}$ (Hahn et al. 2002), i.e., 330 times smaller than the estimated upper limit of warm dust in HD 38529.

\section{CONCLUSIONS}

HD 38529 harbors a planetary system consisting of at least two planets (with $M \sin i$ of 0.8 and $12.2 M_{\text {Jup }}$, semimajor axes of 0.13 and $3.74 \mathrm{AU}$, and eccentricities of 0.25 and 0.35 ) and a likely population of dust-producing planetesimals that are responsible for the $70 \mu \mathrm{m}$ excess emission detected by Spitzer. Using analytical and numerical dynamical analysis, in this paper we have constrained the distribution of the potential dust-producing planetesimals from the study of the dynamical perturbations of the two known planets, considering in particular the effect of secular resonances. A dust disk inner edge at 5.5 AU would naturally arise from the gravitational scattering of planetesimals and dust grains by the outermost planet. We show that larger inner cavities in the dust disk, which would be consistent with the observed SED, can be created due to the secular effects that arise from the interaction between the two massive planets. From the analysis of the secular perturbations we identify three regions that could harbor planetesimal populations in low-eccentricity orbits (where the planetesimals could be long-lived): $0.4-0.8,20-50$, and beyond $60 \mathrm{AU}$. From the modeling of the SED we conclude that the planetesimals responsible for most of the dust emission observed by Spitzer are likely located within the 20-50 AU region. In this regard, HD 38529 resembles the configuration of the solar system's Jovian planets + Kuiper Belt. The SED models give a dust mass estimate of $(1-5) \times 10^{-10} M_{\odot}$ of $10 \mu \mathrm{m}$ particles. The presence of a significant population of small grains inside $100 \mathrm{AU}$ [with the particle size distribution following $n(b) \propto b^{-3.5}$, and with $b_{\min }=2 \mu \mathrm{m}$ and $\left.b_{\max }=10 \mu \mathrm{m}\right]$ is excluded to a $3 \sigma$ certainty level based on the lack of a significant continuum emission at $\lambda<30 \mu \mathrm{m}$. We do not find evidence of dust emission within the innermost region, with a $3 \sigma$ dust mass upper limit of $10^{-7} M_{\oplus}$ (in $10 \mu \mathrm{m}$ grains), suggesting any remnant dust belt would have a mass smaller than 330 times that in the solar system's zodiacal cloud.

The SED models are degenerate. We need to break this degeneracy to get a better understanding of this planetary system, in particular, of how the spatial distribution of the dust and the planetesimals is affected by the gravitational perturbations of the two planets. This requires spatially resolved images to constrain the disk sizes, high-resolution spectroscopy observations to look for spectral features that could constrain the grain size and composition, and/or accurate photometric points in the $33-70 \mu \mathrm{m}$ range and in the submillimeter to better determine the shape of the SED.

We thank the rest of the FEPS team members, colleagues at the Spitzer Science Center, and members of all the Spitzer instrument teams for advice and support. This work is based (in part) on observations made with the Spitzer Space Telescope, which is operated by the Jet Propulsion Laboratory, California Institute of Technology, under NASA contrast 1407. A. M.-M. is under contract with the Jet Propulsion Laboratory (JPL), funded by NASA through the Michelson Fellowship Program. JPL is managed for NASA by the California Institute of Technology. A. M.-M. is also supported by the Lyman Spitzer Fellowship at Princeton University. R. M. and M. R. M. are supported in part through the LAPLACE node of NASA's Astrobiology Institute. R. M. also acknowledges support from NASA's Origins of Solar Systems research program. S. W. was supported through DFG Emmy Noether grants WO $875 / 2-1$ and WO $875 / 2-2$. FEPS is pleased to acknowledge support from NASA contracts 1224768 and 1224566, administered through JPL.

\section{REFERENCES}

Ardila, D. R., Golimowski, D. A., Krist, J. E., Clampin, M., \& Williams, J. P. 2004, ApJ, 617, L147

Artymowicz, P., Burrows, C., \& Paresce, F. 1989, ApJ, 337, 494

Backman, D. E., Dasgupta, A., \& Stencel, R. E. 1995, ApJ, 450, L35

Backman, D. E., \& Paresce, F. 1993, in Protostars and Planets III, ed. E. H. Levy \& J. I. Lunine (Tucson: Univ. Arizona Press), 1253

Barnes, R., \& Raymond, S. N. 2004, ApJ, 617, 569

Beichman, C. A., et al. 2005a, ApJ, 622, 1160

. 2005b, ApJ, 626, 1061

Bryden, G., et al. 2006, ApJ, 636, 1098

Burns, J. A., Lamy, P. L., \& Soter, S. 1979, Icarus, 40, 1

Butler, R. P., et al. 2006, ApJ, 646, 505

Dent, W. R. F., Walker, H. J., Holland, W. S., \& Greaves, J. S. 2000, MNRAS, 314,702

Dermott, S. F., et al. 2002, in Proc. Asteroids, Comets, Meteors, ed. B. Warmbein (ESA SP-500; Noordwijk; ESA), 319

Dominik, C., \& Decin, G. 2003, ApJ, 598, 626

Duncan, M., Quinn, T., \& Tremaine, S. 1989, Icarus, 82, 402

Fischer, D. A., et al. 2001, ApJ, 551, 1107

2003, ApJ, 586, 1394

Gonzalez, G., et al. 2001, AJ, 121, 432

Greaves, J. S., Holland, W. S., Wyatt, M. C., Dent, W. R. F., \& Robson, E. I. 2005, ApJ, 619, L187

Greaves, J. S., Mannings, V., \& Holland, W. S. 2000, Icarus, 143, 155

Greaves, J. S., et al. 1998, ApJ, 506, L133

Hahn, J. M., Zook, H. A., Cooper, B., \& Sunkara, B. 2002, Icarus, 158, 360 Holland, W. S., et al. 2003, ApJ, 582, 1141

Jura, M., Ghez, A. M., White, R. J., McCarthy, D. W., Smith, R. C., \& Martin, P. G. 1995, ApJ, 445,451

Jura, M., et al. 2004, ApJS, 154, 453

Kalas, P., Graham, J. R., \& Clampin, M. 2005, Nature, 435, 1067

Kalas, P., Graham, J. R., Clampin, M., \& Fitzgerald, M. P. 2006, ApJ, 637, L57

Krivov, A. V., Mann, I., \& Krivova, N. A. 2000, A\&A, 362, 1127

Li, A., \& Greenberg, J. M. 1998, A\&A, 331, 291

Li, A., \& Lunine, J. 2003, ApJ, 590, 368

Liou, J. C., \& Zook, H. A. 1999, AJ, 118, 580

Liu, M. C. 2004, Science, 305, 1442

Malhotra, R. 1998, in ASP Conf. Ser. 149, Solar System Formation and Evolution, ed. D. Lazzaro et al. (San Francisco: ASP), 37

Metchev, S. A., Eisner, J. A., Hillenbrand, L. A., \& Wolf, S. 2005, ApJ, 622, 451 Meyer, M. R., et al. 2006, PASP, 118, 1690 
Moro-Martín, A., \& Malhotra, R. 2002, AJ, 124, 2305

2003, AJ, 125, 2255

2005, ApJ, 633, 1150

Moro-Martín, A., Wolf, S., \& Malhotra, R. 2005, ApJ, 621, 1079

Moro-Martín, A., et al. 2007, ApJ, 658, 1312

Murray, N., \& Dermott, S. 1999, Solar System Dynamics (Cambridge: Cambridge Univ. Press)

Perryman, M. A. C., et al. 1997, A\&A, 323, L49

Raymond, S. N., Barnes, R., \& Kaib, N. A. 2006, ApJ, 644, 1223

Roques, F., Scholl, H., Sicardy, B., \& Smith, B. A. 1994, Icarus, 108, 37

Stapelfeldt, K. R., et al. 2004, ApJS, 154, 458
Stern, S. A. 1996, AJ, 112, 1203

Telesco, C. M., et al. 2005, Nature, 433, 133

Valenti, J. A., \& Fischer, D. A. 2005, ApJS, 159, 141

Weingartner, J. C., \& Draine, B. T. 2001, ApJ, 548, 296

Wilner, D. J., Holman, M. J., Kuchner, M. J., \& Ho, P. T. P. 2002, ApJ, 569, L115

Wisdom, J., \& Holman, M. 1991, AJ, 102, 1528

Wolf, S., \& Hillenbrand, L. A. 2003, ApJ, 596, 603

Wyatt, M. C. 2005, A\&A, 433, 1007

Wyatt, M. C., Smith, R., Greaves, J. S., Beichman, C. A., Bryden, G., \& Lisse, C. M. 2007, ApJ, 658, 569 